\title{
Multi-path and Multi-hop Energy Efficient Routing in Wireless Sensor Network
}

\author{
Umesh Kumbhalkar \\ Research Scholar \\ Department of Computer Science \& Application \\ RKDF University, Bhopal, India
}

\author{
Sharad Gangele, $\mathrm{PhD}$ \\ Professor \\ Department of Computer Science \& Application \\ RKDF University, Bhopal, India
}

\begin{abstract}
Wireless sensor networks have strict constrains in size, weight and energy. The conflict of long lifetime and strict energy constrain brings a natural integration of energy harvesting into wireless sensor networks. A modified low energy adaptive clustering hierarchy $(\mathrm{LEACH})$ protocol has been proposed to increase the network life time. Modified LEACH is divided into two main phases at the first setup phase and at the second steady state phase. Setup phase has been modified such that cluster head (CH) selection will be selected only from advanced nodes. Results showed significant improvement in WSN overall lifetime. Simulations show number of dead nodes vs rounds. Comparison between pure LEACH and modified one showed significant improvement in lifetime, also comparison between modified LEACH and multi-path multi-hop energy efficient routing protocol shows significant improvement in performance.
\end{abstract}

\section{General Terms}

Wireless Sensor Network, Energy Efficiency

\section{Keywords}

Wireless Sensor Network, Energy Efficiency, LEACH, Multi-path, Multi-hop.

\section{INTRODUCTION}

A wireless sensor network (WSN) contain thousands of assembled sensors configured into a cooperative network [1, 2]. Sensors nodes together monitor physical phenomena, such as temperature, humidity, sound, motion, heat, vibrations, or pressure. Nodes usually sense, process information and communicate with their neighbors wirelessly. WSN has become an indispensable part of IoT, which is composed of several sensors to sense physical data from the environment. The sensed data are processed, stored, and transmitted by the sensor and communicate with a user or observer via Internet as presented in Figure 1. Wireless sensor networks are informationretrieval networks [3, 4]. Basically, nodes cooperate to aggregate the information of data and deliver it to monitoring terminal(s), called sink(s). Wireless sensor networks are used in various industrial and civilian applications, such as agriculture application, environmental monitoring, security and surveillance and smart building [5].

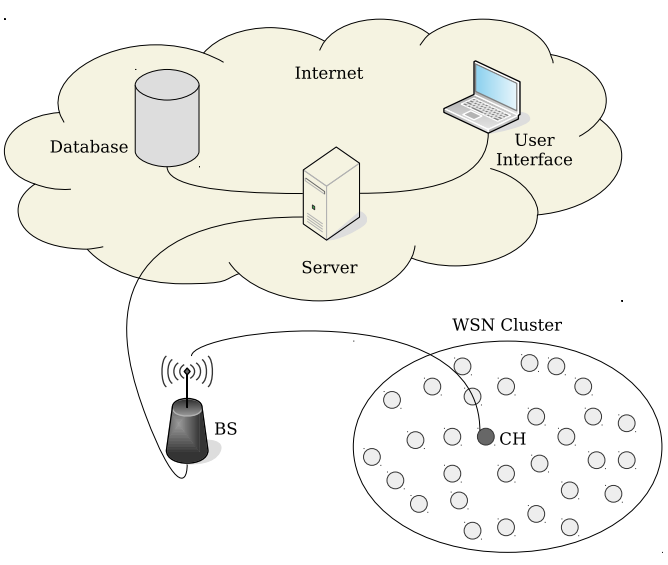

Fig. 1. Basic WSN Model

All over the world, WSNs have received tremendous attention in the past years, from both academia and industry. A huge amount of research activities have been done to explore and solve various design and application issues, and significant advances have been made in the development and deployment of WSNs [6, 7, 8]. Despite the countless applications of WSNs, these networks have some severe restrictions, such as limited battery power, limited transmission range, and limited storage. These constraints have raised some interesting challenges that must be addressed and solved while designing a routing algorithm. The algorithm must be energy efficient and scalable. Moreover, the energy resources for each sensor node must be managed effectively. Naturally, gathering sensor nodes into clusters has been extremely adopted by researchers to achieve the required scalability and attain high energy efficiency and prolong network lifetime in large-scale WSN environments [9]. The main process in any cluster-based algorithm is to choose a set of cluster heads and partition the remainder of nodes into clusters. Under common clustering architecture, nodes within a cluster transmit data to their cluster head which in turn conveys the aggregated data to the base station or relays it to other heads adjacent to the base station. Normally, cluster heads expend more energy compared to non-cluster head nodes. Cluster heads closer to the base station consume their energy faster than other nodes due to the aggregated relay traffic sent from far away heads. To address this, a novel network model is developed that aims to shorten 
the long-distance communications for the sake of maximizing the network lifetime in this research. Precisely, there are more clusters at the location closer to the base station. Thus, they will dissipate less energy during the intra-cluster communication and can maintain more energy to handle the high traffic received from far away clusters [10].

The WSN nodes operate on battery power. However, onboard energy storage has very limited capacity to sustain WSN nodes for long time period due to their strict size and weight constrains. Therefore the energy consumption of WSN nodes and balance of the network energy is an important factor. WSN lifetime is one of the most major issues that should be taken into consideration while designing or adopting network protocols. Network lifetime in homogeneous networks less than heterogeneous networks. In standard low energy adaptive clustering hierarchy protocol (LEACH) cluster heads $(\mathrm{CH})$ randomly and independently are selected. Mainly cluster heads consume more power than other normal nodes because most $\mathrm{CH}$ power goes in data aggregation from other nodes in cluster and resend to sink node or base station. Here introduced modification to Setup phase in standard LEACH which will lead to significant improvement. As a result network lifetime has been increased when proposed modified LEACH had been used. It will be more efficient if cluster heads chosen from advanced nodes only that will increase $\mathrm{CH}$ lifetime and the whole network as well. Thats why the idea of modified LEACH raised up.

\section{RELATED WORK}

Heinzelman et al. [11] proposed LEACH and centralized LEACH (LEACH-C) [12] to solve the problem of energy load balancing by rotating the role of $\mathrm{CHs}$ in round-robin fashion. Each round in LEACH divides clustering process into two phases: set-up phase and steady phase. During the set-up phase, each node generates a random number between 0 and 1 . Thus it becomes a $\mathrm{CH}$ if the random number is less than a threshold $T(n)$, given by:

$$
T(n)= \begin{cases}\frac{P}{1-P\left(r \quad \bmod \frac{1}{P}\right)} & \text { if } n \in G \\ 0 & \text { otherwise }\end{cases}
$$

where $P$ is the percentage of $\mathrm{CHs}$ being elected across all clusters, $r$ is the current round, $G$ is the set of nodes that have not been elected as $\mathrm{CHs}$ for the past $1 / P$ rounds and $n$ is the nodes.

Taj et al. [13] proposed ICH-LEACH protocol, after conducting a research on LEACH protocol, they came up with an improved protocol. In fact, this protocol is characterized by the adjustment of the power of transmission, and the distance between the base station and cluster heads. This protocol surpassed the original one in term of energy consumption, network lifetime and the number of sand data by avoiding a direct send of data if the distance from the sink is too far. The simulation made with initial energy for every node shows that proposed algorithm is efficient compared to the original one.

ICH-LEACH protocol aims to reduce the energy consumption in order to extend the network lifetime and send more data to the sink. Also, its proposition uses the exact phases of LEACH, and use the same number of cluster head as LEACH. Actually, the main problem of LEACH is to send Data from the cluster-Head to the sink directly. Thus, the necessary energy for transmitting Data is high. Even more, if the sink is too far the transmission is impossible and the data is lost. So, by using an intermediate cluster-head if the distance is far, then it make sure that the amount of consumed energy is less during each round and the amount of transmitted.
Alharthi et al. [14] proposed LEACH (HT2HL), the hybrid threshold sensitive and two-level heterogeneous protocol in their research. The performance of the proposed protocol outperforms DEEC and SEP as presented in the simulation result. The drawbacks in LEACH are dealt with in their work. For instance, SNs can communicate directly with the BS if all CHs are far from them and they adjust their transmission power based on the distance from $\mathrm{CHs}$ and the BS. Thresholds are incorporated to reduce the number of transmission which conserve energy. The work is still in progress to improve the performance by balancing the cluster size and considering multihop communication for farthest $\mathrm{CHs}$.

Arumugam et al. [15] presented EE-LEACH, development of energy-efficient LEACH Protocol for data gathering in WSN, to improve the lifetime of the sensor network. The coverage probability is derived with respect to the Gaussian distribution. A sorting algorithm based on the residual energy of the neighbor nodes is executed to obtain the list of neighbor nodes. Data ensemble also takes place while aggregating the nodes. Data ensemble can save considerable energy while the source nodes forming one cluster are deployed in a relatively small area when the sink node is far away from the source nodes. An election weight is determined by taking account of the concentration degree of SNs and their residual energy for optimal CH election. The EE-LEACH Protocol results in a better packet delivery ratio, lesser energy consumption and lesser E2E delay than the EBRP and LEACH Protocols. The experimental results shows that the proposed EE-LEACH yields better outcomes than the existing EBRP and LEACH Protocols.

An efficient-energy-aware routing protocol is mandatory for data gathering. All the sensor nodes have similar significance and equal capabilities. This motivates the need for improving the lifetime of the sensor nodes and sensor network. The objective of the proposed EE-LEACH Protocol is to reduce the energy consumption and increase the network longevity. Here, Gaussian distribution model is used for effective coverage of the sensing network area.

Al-Sodairi et al. [16] proposed a new extended LEACH-based clustering algorithm to enhance WSN performance in terms of reliability, energy efficiency, and lifetime. First, their algorithm, EMLEACH, supports new rules for cluster-head selection and round time computing based on the remaining energy. Second, EMLEACH improves the communication model from single-hop to multi-hop between $\mathrm{CHs}$ and sink node using two operating processes: leveling and generic multi-hop routing. The Castalia simulator was used to evaluate the performance of the proposed algorithm, EM-LEACH, compared to LEACH and MR-LEACH. The performance evaluation included different aspects related to network reliability, energy efficiency, and lifetime. The simulation results proved that the performance improvement of EM-LEACH algorithm, in terms of packet delivery, is extremely good compared to LEACH and MR-LEACH. Additionally, EM-LEACH provided around $16 \%$ to $25 \%$ extra network lifetime compared to LEACH and presented results similar to MR-LEACH.

Kumbhalkar et al. [17] proposed, "TBEEDRA (Threshold-Based Energy-Estimated Distributed Routing Algorithm)" in wireless sensor network as a reactive network routing protocol with considering three different levels of sensor node heterogeneity. TBEEDRA combines the best features of EDDEEC protocol and energy level evaluation method. Due to the concept of energy level based cluster head selection, hard and soft threshold value, three levels of node heterogeneity and being reactive routing network protocol TBEEDRA produces increase in energy efficiency, enhanced lifetime of network and also maximum throughput as shown in the simulation result. In comparison with DEEC, DDEEC, EDEEC and EDDEEC with the proposed strategy of TBEEDRA, it can be con- 
cluded that the protocol TBEEDRA will perform well in small as well as large geographical networks and best suited for time critical applications. Finally, in future, the concept and implementation of the mobile base station can be introduced in TBEEDRA to perform the next level of advanced technology of wireless sensor network due to three levels of heterogeneity and being reactive routing network protocol, so it produces increased level in energy efficiency and enhanced network lifetime.

Sarkar et al. [18] proposed a novel approach for increasing lifetime of the network. The proposed protocol for wireless sensor network is a modification of LEACH protocol. The lifetime of the network has improved by more than $40 \%$. Lifetime of the network is directly proportional to energy available in the nodes. In this work, selection of cluster head is depending on maximum residual energy so cluster head can able to serve for more number of rounds. Preserving energy of nodes is achieved by avoiding cluster formation in each and every round. Formation of cluster is dependent on the threshold value. It saves energy required for creating a new cluster and also increases the lifetime of the network.

Most of the existing works concentrate on best cluster head selection in each round, which leads to some amount of energy consumption. In this work, they have tried to avoid cluster formation in each round so that they saved energy required for making cluster or setup phase. Heterogeneous energy level is assigned to some of the sensor nodes, and it helps in preserving energy. This can be achieved by avoiding cluster formation as much as possible throughout the network lifetime. There are two phases of LEACH protocolsetup phase and steady-state phase. In this proposed solution, changes are made in setup phase only.

\section{ENERGY MODEL}

For the energy calculation, a simple model shown in Figure 2 is used.

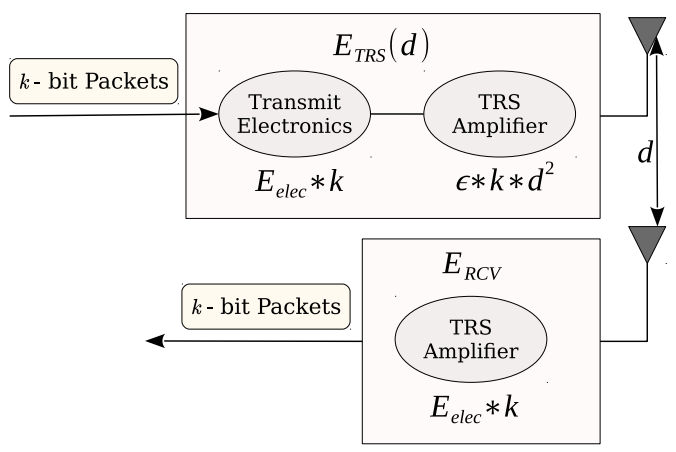

Fig. 2. Energy Model of Wireless Sensor Network

The packet length assumed to be $k$-bits where the radio dissipates $E_{\text {elec }}=50 \mathrm{~nJ} / \mathrm{bit}$ to run the transmitter or receiver circuitry and $E a m p=100 \mathrm{pJ} / \mathrm{bit} / \mathrm{m}^{2}$ for the transmit amplifier to achieve an acceptable $E_{b} / N_{o}$. It is also assume a loss factor $r^{2}$ due to channel transmission [19, 20]. Thus, using the radio model the energy spends by any transmitter to send a $k$-bit message over a distance $d$ is $E_{T x}(k, d)$ :

$$
\begin{aligned}
& E_{T x}(k, d)=E_{T x-\text { elec }}(k)+E_{T x-a m p}(k, d) \\
& E_{T x}(k, d)=k \times E_{\text {elec }}+k \times E_{\text {amp }} \times d^{2}
\end{aligned}
$$

The first term of (1) represents the energy consumption of radio dissipation, while the second represents the energy consumption for amplifying radio. The electronics energy $E_{\text {elec }}$ depends on factors such as the digital coding, modulation, filtering, and spreading of the signal. And to receive this message, the radio expends $E_{R x}(k)$ :

$$
E_{R x}(k)=E_{R x-e l e c}(k)=k \times E_{\text {elec }}
$$

After sensor nodes deployment, nodes decide independently whether to be a cluster head or not by generating a random number between 0 and 1 and then compares the threshold value. Here in modified LEACH threshold value calculated by:

$$
T(n)= \begin{cases}\frac{P \times a}{1-a \times P\left[r \bmod \left(\frac{1}{P}, a\right)\right]} & \text { if } n \in G \\ 0 & \text { otherwise }\end{cases}
$$

By this way $\mathrm{CH}$ will be chosen from advanced nodes mostly.

\section{PROPOSED MODEL}

In earlier works, one of the main assumptions is that energy is only consumed for the packet transmission, not packet sensing and processing operations in the node.

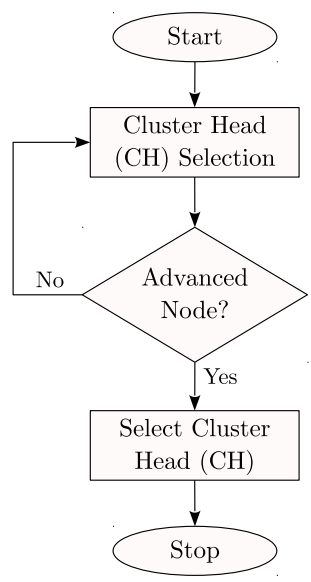

Fig. 3. Workflow of Proposed Model

\section{Algorithm of Proposed Model:}

Deploy the sensor nodes randomly across the network area.

for all sensor nodes do $i=1$ to $n, S(i)=\left(X_{i}, Y_{i}\right)$

Randomly establish the sensor nodes.

end for

if

Node $==$ Advanced Node

Select $\mathrm{CH}$

end if

for every cluster

Transmit the sensed data to the $\mathrm{CH}$.

end for

$\mathrm{CH}$ forwards it to the sink node.

In this work, the main contribution is considered as energy consumption not only for data transmission but also for node electronics, i.e., data sensing-processing-storing in an energy harvesting wireless sensor.

The proposed model is divided into two main phases at the first setup phase and at the second steady state phase. In the first phase 
nodes are spread randomly allover network area and cluster heads should be elected randomly [21, 22]. The selection of cluster head in the setup phase of the proposed model is presented in Figure 3 This algorithm presents the function of the proposed model. Cluster head $(\mathrm{CH})$ is selected if it is advanced node for higher energy efficiency and longer network lifetime.

\subsection{Simulation and Result}

In this paper the term of dead nodes is introduced. Dead node can be defined as the node which empty battery that cannot send or receive any data. A simulation for dead nodes against round number also showed significant improvement in network lifetime as shown in Figure 4 as most power consumption is going in cluster heads as previously declared.

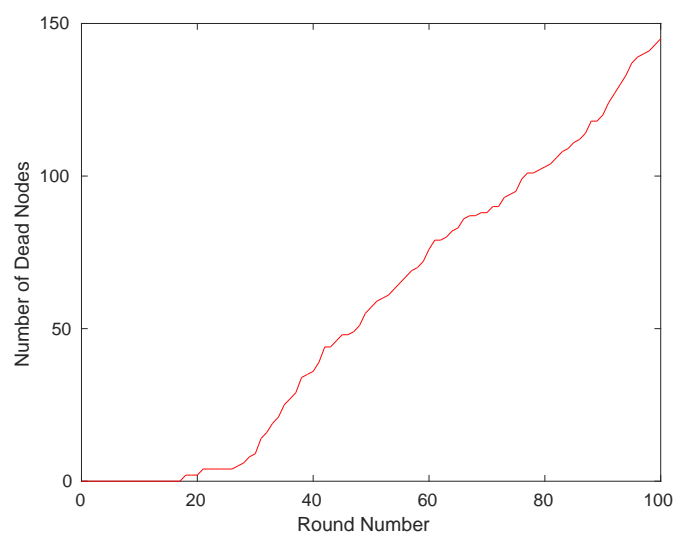

Fig. 4. Number of Dead Nodes during Rounds in LEACH

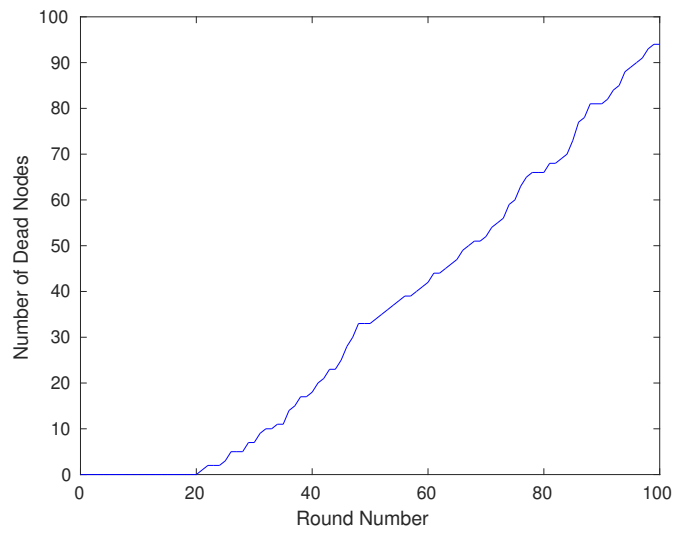

Fig. 5. Number of Dead Nodes during Rounds in Proposed Protocol

Simulations has been done with the above mentioned assumptions and here in Figure 6 shown average energy per node which shows significant improvement in energy consumption against round numbers which leads to overall network lifetime improvement as well.

Taking into consideration that all these simulations has been made with assumption that heterogeneous nodes percentage is $10 \%$ of

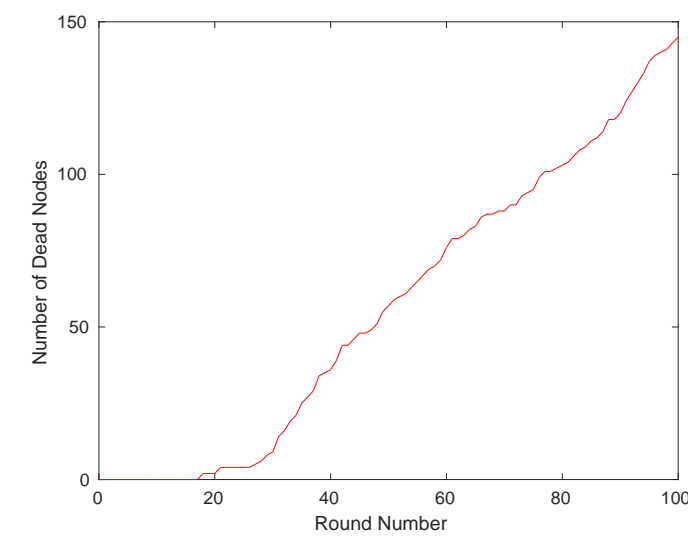

Fig. 6. Average Energy of Each Node in LEACH

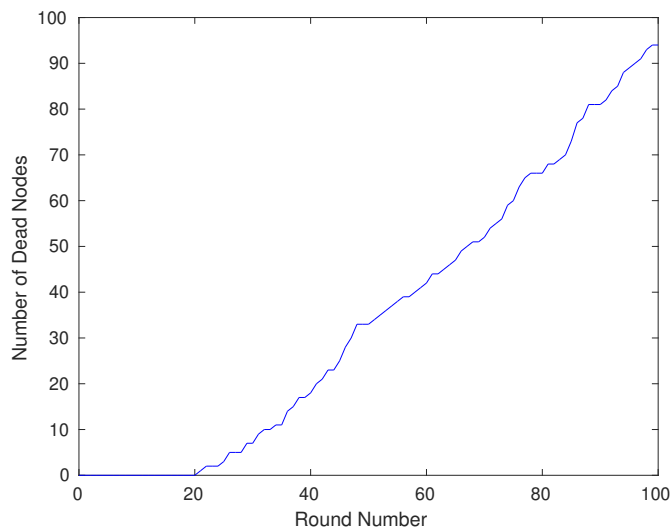

Fig. 7. Average Energy of Each Node in Proposed Protocol

total number of nodes. Here a major question can be raised, what if number of heterogeneous nodes has been increased? And how it will affect over all network lifetime, shown in figure improvement in average energy and dead nodes vs round number.

As shown in Figure 5 improvement when number of advanced nodes increase is not effective because the main number which affects here is percentage of cluster head entire network not the percentage of advanced nodes only.

Also a comparison between Multi-path Multi-hop Energy Efficient Routing protocol and modified LEACH has been done in Figure 6 , Figure 7 and as shown in figures a significant improvement is achieved in average energy per node and total number of dead nodes when cluster heads chosen from heterogeneous nodes only. The proposed protocol is better than both standard LEACH.

\section{CONCLUSION}

Due to the limited amount of energy in the sensor nodes, the analysis of the energy consumption of the WSN is very important. For this reason, a modified LEACH routing protocol has been developed for WSN. Modified LEACH is separated into two main phases 1) Setup phase 2) Steady state phase. Setup phase has been modified such that $\mathrm{CH}$ selection will be selected only from advanced nodes. From the investigative analysis and the comparative 
results, the conclusion comes that modified LEACH of Heterogeneous WSN provides a significant improvement and good performance in terms of energy consumption and increasing the level in lifetime of the heterogeneous wireless sensor networks than in standard LEACH and proposed systems.

The energy consumption analysis of wireless sensor network shows the significance of powering nodes from non-renewable energy sources, such as batteries. However this method of powering cannot satisfy all of the simultaneous demands for long life, low volume, low weight and limited environmental impact. Wireless sensor networks can be powered by environmentally scavenged energy.

\section{References}

[1] K. Sohraby, D. Minoli, and T. Znati, Routing Protocols for Wireless Sensor Networks. Wiley, 2007. [Online]. Available: https://doi.org/10.1002/9780470112762.ch6

[2] N. Alaoui, J. Cances, and V. Meghdadi, "Energy consumption in wireless sensor networks for network coding structure and arq protocol," in 2015 International Conference on Electrical and Information Technologies (ICEIT), March 2015, pp. 317-321. [Online]. Available: https://doi.org/10. 1109/EITech.2015.7162963

[3] S. Misra and S. Goswami, Routing in Wireless Sensor Networks. Wiley, 2014. [Online]. Available: https://doi.org/ 10.1002/9781119114864.ch11

[4] V. Rajaravivarma and and, "An overview of wireless sensor network and applications," in Proceedings of the 35th Southeastern Symposium on System Theory, 2003., March 2003, pp. 432-436. [Online]. Available: https: //doi.org/10.1109/SSST.2003.1194607

[5] K. Sohraby, D. Minoli, and T. Znati, Applications of Wireless Sensor Networks. Wiley, 2007. [Online]. Available: https://doi.org/10.1002/9780470112762.ch2

[6] K. A. Darabkh and O. A. Alsukour, "Novel protocols for improving the performance of odmrp and eodmrp over mobile ad hoc networks," Int. J. Distrib. Sen. Netw., vol. 2015, pp. 174:174-174:174, Jan. 2016. [Online]. Available: https://doi.org/10.1155/2015/348967

[7] R. T. Al-Zubi, M. Krunz, G. Al-Sukkar, M. Hawa, and K. A. Darabkh, "Packet recycling and delayed ack for improving the performance of tcp over manets," Wireless Personal Communications, vol. 75, no. 2, pp. 943963, Mar 2014. [Online]. Available: https://doi.org/10.1007/ s11277-013-1401-8

[8] K. A. Darabkh, W. Y. Albtoush, and I. F. Jafar, "Improved clustering algorithms for target tracking in wireless sensor networks," The Journal of Supercomputing, vol. 73, no. 5, pp. 1952-1977, May 2017. [Online]. Available: https: //doi.org/10.1007/s11227-016-1898-1

[9] K. A. Darabkh, W. S. Al-Rawashdeh, R. T. Al-Zubi, and S. H. Alnabelsi, "C-dtb-chr: centralized density- and thresholdbased cluster head replacement protocols for wireless sensor networks," The Journal of Supercomputing, vol. 73, no. 12, pp. 5332-5353, Dec 2017. [Online]. Available: https://doi.org/10.1007/s11227-017-2089-4
[10] K. A. Darabkh, N. J. Al-Maaitah, I. F. Jafar, and A. F. Khalifeh, "Ea-crp: A novel energy-aware clustering and routing protocol in wireless sensor networks," Computers \& Electrical Engineering, vol. 72, pp. 702-718, 2018. [Online]. Available: http://www.sciencedirect.com/science/ article/pii/S0045790617307644

[11] W. R. Heinzelman, A. Chandrakasan, and H. Balakrishnan, "Energy-efficient communication protocol for wireless microsensor networks," in Proceedings of the 33rd Annual Hawaii International Conference on System Sciences, Jan 2000, pp. 10 pp. vol.2-. [Online]. Available: https://doi.org/10.1109/HICSS.2000.926982

[12] W. B. Heinzelman and A. P. Chandrakasan and H. Balakrishnan, "An application-specific protocol architecture for wireless microsensor networks," IEEE Transactions on Wireless Communications, vol. 1, no. 4, pp. 660-670, Oct 2002. [Online]. Available: https://doi.org/10.1109/TWC. 2002.804190

[13] M. B. M. Taj and M. A. Kbir, "Ich-leach: An enhanced leach protocol for wireless sensor network," in 2016 International Conference on Advanced Communication Systems and Information Security (ACOSIS), Oct 2016, pp. 1-5. [Online]. Available: https://doi.org/10.1109/ACOSIS.2016.7843949

[14] S. A. Alharthi and P. A. Johnson, "Threshold sensitive heterogeneous leach protocol for wireless sensor networks," in 2016 24th Telecommunications Forum (TELFOR), Nov 2016, pp. 1-4. [Online]. Available: https://doi.org/10.1109/ TELFOR.2016.7818743

[15] G. S. Arumugam and T. Ponnuchamy, "Ee-leach: development of energy-efficient leach protocol for data gathering in wsn," EURASIP Journal on Wireless Communications and Networking, vol. 2015, no. 1, p. 76, Mar 2015. [Online]. Available: https://doi.org/10.1186/s13638-015-0306-5

[16] S. Al-Sodairi and R. Ouni, "Reliable and energy-efficient multi-hop leach-based clustering protocol for wireless sensor networks," Sustainable Computing: Informatics and Systems, vol. 20, pp. 1-13, 2018. [Online]. Available: https://doi.org/10.1016/j.suscom.2018.08.007

[17] U. Kumbhalkar and S. Gangele, "Threshold-based energyestimated distributed routing algorithm in wireless sensor network," International Journal of Scientific Research in Science, Engineering and Technology (IJSRSET), vol. 04, no. 09, pp. 149-158, 2018. [Online]. Available: http: //ijsrset.com/\%20IJSRSET184916

[18] P. Sarkar and C. Kar, "Th-leach: Threshold value and heterogeneous nodes-based energy-efficient leach protocol," in Algorithms and Applications, S. K. Das and N. Chaki, Eds. Singapore: Springer Singapore, 2018, pp. 41-49.

[19] M. Abo-Zahhad, M. Farrag, A. Ali, and O. Amin, "An energy consumption model for wireless sensor networks," in 5th International Conference on Energy Aware Computing Systems Applications, March 2015, pp. 1-4. [Online]. Available: https://doi.org/10.1109/ICEAC.2015.7352200

[20] Y. M. Kadioglu, "Energy consumption model for data processing and transmission in energy harvesting wireless sensors," in Computer and Information Sciences, T. Czachórski, 
E. Gelenbe, K. Grochla, and R. Lent, Eds. Cham: Springer International Publishing, 2016, pp. 117-125.

[21] A. Amwary, D. Maga, and T. Nahdi, "Modified leach protocol for heterogeneous wireless networks," in 2016 New Trends in Signal Processing (NTSP), Oct 2016, pp. 1-4. [Online]. Available: https://doi.org/10.1109/NTSP.2016.7747774

[22] F. Zhao, Y. Xu, R. Li, and W. Zhang, "Improved leach communication protocol for wsn," in 2012 International Conference on Control Engineering and Communication Technology, Dec 2012, pp. 700-702. [Online]. Available: https://doi.org/10.1109/ICCECT.2012.60 